\title{
Spin-orbit effects at chiral surfaces
}

\author{
N. K. Lewis, ${ }^{1,2}$ P. J. Durham, ${ }^{3}$ W. R. Flavell, ${ }^{1}$ and E. A. Seddon ${ }^{1,2}$ \\ ${ }^{1}$ School of Physics and Astronomy and the Photon Science Institute, University of Manchester, Oxford Road, Manchester, M13 9PL, UK \\ ${ }^{2}$ The Cockcroft Institute, Daresbury Laboratory, Sci-Tech Daresbury, Warrington, WA4 4AD, UK \\ ${ }^{3}$ Daresbury Laboratory, Sci-Tech Daresbury, Warrington, WA4 4AD, UK
}

(Received 22 May 2018; published 18 June 2018)

\begin{abstract}
Two-dimensional hexagonal and oblique lattices were investigated theoretically with the aim of observing differences in the spin expectation values between chiral and achiral systems. The spin-resolved band structures were derived from the energy eigenvalues and eigenfunctions of a Hamiltonian that includes the lattice potential and the spin-orbit interaction. The spin texture of the achiral hexagonal system was shown to have two nonzero components of the spin polarization, whereas all three components were calculated to be nonzero for the chiral system. The longitudinal component, found to be zero in the achiral lattice, was observed to invert between the enantiomorphs of the chiral lattice. A heuristic model was introduced to discern the origin of this inverting spin polarization by considering the dynamics of an electron in chiral and achiral lattices. This model was further used to demonstrate the change in magnitude of the spin polarization as a function of the lattice parameters and an electric field perpendicular to the lattice.
\end{abstract}

DOI: 10.1103/PhysRevB.97.235427

\section{INTRODUCTION}

Symmetry-breaking systems have been extensively studied and shown to produce novel spin-polarization effects $[1,2]$. Experimentally, spin- and angle-resolved photoemission have been used to determine the spin density of two-dimensional systems which lack inversion symmetry [3,4]. Examples of this include the Rashba effect in $\mathrm{Au}(111)$ [5], the giant Rashba effect in $\mathrm{Bi} / \mathrm{Ag}(111)$ [6-10], and the spin-valley polarization present in transition-metal dichalcogenides such as $\mathrm{WSe}_{2}$ [11-13]. Such phenomena are important for spintronic devices $[14,15]$. However, the spin texture of two-dimensional chiral systems (those that lack mirror symmetry) has had limited study.

In electron-scattering experiments involving gas-phase chiral molecules, spin polarization inversion has been demonstrated. In particular, Mayer et al. showed [16] that longitudinally spin-polarized electrons transmitted through randomly oriented and enantiomerically pure chiral molecules produce an energy-dependent intensity asymmetry between parallel and antiparallel spin polarized electrons. They further observed that the intensity asymmetry "mirrored" between the enantiomers. In another scattering experiment, electrons propagating parallel to the helical axis of DNA have been shown to develop a longitudinal spin polarization independent of the initial spin orientation [17,18], observations that have been associated with spin filtering effects [19]. These electron-scattering experiments (involving systems that lack mirror symmetry) and the electron-spin polarization show a correlation that has been the subject of theoretical investigations [20,21].

The main underlying phenomenon that contributes to all these effects is the spin-orbit interaction. Rashba et al. considered the spin-orbit interaction in two-dimensional electron-gas systems [22]. In the original Rashba model the electronic motion is assumed to be confined to, but free in, the $x-y$ plane and affected by the spin-orbit interaction, where the potential gradient is in the $z$ direction only and approximated as a constant. This model can be solved analytically, and two important results arise. First, the two-dimensional energy bands are parabolic but spin split by an amount proportional to the Rashba parameter $\alpha$, which is determined by the spin-orbit coupling strength. Second, the spin polarization of each band is entirely in the $x-y$ plane and orthogonal to the surface crystal momentum $k_{\|}$; there is no longitudinal or perpendicular spin polarization. This model has been used as a phenomenological guide to interpreting experimental results [8] for systems with a relatively-free-electron-like band structure. The most rigorous approach currently available is to use fully self-consistent relativistic density-functional methods [23]. Such calculations are demanding but can produce good agreement with experiment. However, as Premper et al. have suggested [24], useful insight can be gained by a simplified approach. For example, they used a semirelativistic Hamiltonian with a potential describing the structural symmetry of $\mathrm{Bi} / \mathrm{Ag}(111)$ to reveal key features of the spin-polarized band structure (which were observed in photoemission experiments [6]) and the essence of the effects of the spin-orbit interaction. This is not the only method that can be used to extend the original Rashba model. $\boldsymbol{k} \cdot \boldsymbol{p}$ theory was used to successfully model hexagonal warping effects in $\mathrm{Bi}_{2} \mathrm{Ti}_{3}$ [25].

Presented here is a semirelativistic model that builds upon the work conducted by Premper et al. by focusing on twodimensional lattices with and without mirror symmetry. There are two types of Bravais lattice considered here: the hexagonal lattice, an achiral structure, and the oblique lattice, the only two-dimensional Bravais lattice to lack mirror symmetry. The results show that, for the hexagonal lattice, there is at least one component of the spin polarization that is always equal to zero (the longitudinal component), which is true for all achiral lattices. In contrast, all three components are in general nonzero 
for a chiral lattice. Furthermore, the spin expectation values are found to invert between the enantiomorphs for the longitudinal component, remain unchanged for the tangential component and differ for the out-of-plane component.

\section{THEORY}

The band structure and spin texture of two-dimensional Bravais lattices are calculated from the eigenvalues and eigenvectors of the semirelativistic Hamiltonian (in Hartree units)

$$
\hat{H}=\frac{\hat{\boldsymbol{p}}^{2}}{2 m^{*}}+\hat{V}+\frac{1}{4 m^{* 2} c^{2}} \hat{\boldsymbol{\sigma}} \cdot(\hat{\boldsymbol{p}} \times \nabla \hat{V}),
$$

where $m^{*}$ is the effective electron mass, $c$ is the speed of light in a vacuum, $\hat{\boldsymbol{p}}$ is the momentum operator, $\hat{\boldsymbol{\sigma}}$ is the vector of Pauli matrices, and $\hat{V}$ is the potential operator [26]. Although the electron motion is confined to the $x-y$ plane, the potential gradient $\nabla V$ spans three-dimensional space with $x, y$, and $z$ components. The potential is a periodic function in the $x-y$ plane described by a Fourier series given by

$$
V(\boldsymbol{r})=\sum_{\boldsymbol{G}} V_{\boldsymbol{G}} e^{i \boldsymbol{G} \cdot \boldsymbol{r}}, \quad \boldsymbol{r}=(x, y),
$$

where $\boldsymbol{G}$ is a two-dimensional reciprocal-lattice vector and the $V_{\boldsymbol{G}}$ are in general a set of complex variables whose properties are described in the supplementary information [27]. This calculation goes beyond the standard Rashba model by including the potential and its in-plane gradients in the $x$ and $y$ directions, which are derived analytically from Eq. (2). The gradient normal to the plane (i.e., in the $z$ direction) is assumed to be a constant given by the standard Rashba parameter:

$$
\alpha=\frac{1}{4 m^{* 2} c^{2}} \frac{\partial V}{\partial z} .
$$

Thus the reciprocal-lattice vectors, the corresponding Fourier components $V_{\boldsymbol{G}}$ and the Rashba parameter $\alpha$ are the principal input parameters to the calculations.

Given a Hamiltonian in the form of Eq. (1) and a periodic potential, it is natural to use a basis formed from the tensor product of momentum eigenkets $|\boldsymbol{k}\rangle$ and spin $\hat{S}_{z}$ eigenkets $\left|m_{s}\right\rangle$. In this basis, the Bloch ket for a given $\boldsymbol{k}$ can be written as

$$
|\Psi(\boldsymbol{k})\rangle=\sum_{\boldsymbol{g}, m_{s}} u_{\boldsymbol{g}, m_{s}}(\boldsymbol{k})\left|\boldsymbol{k}+\boldsymbol{g}, m_{s}\right\rangle,
$$

where $\boldsymbol{g}$ is a reciprocal-lattice vector. The eigenvectors $u_{\boldsymbol{g}, m_{s}}(\boldsymbol{k})$ and the eigenvalues $E(\boldsymbol{k})$ are found by diagonalizing the Hamiltonian matrix in the $\left|\boldsymbol{k}+\boldsymbol{g}, m_{s}\right\rangle$ basis, using the standard techniques of linear algebra [28]. The spin expectation values $\left\langle S_{x, y, z}\right\rangle$ are calculated by constructing the spin density matrix from the full eigenvector $u_{\boldsymbol{g}, m_{s}}(\boldsymbol{k})$ for a given $\boldsymbol{k}$.

This generalized Rashba calculation is a tool with which the parameter space of the model can be easily scanned. The main focus of the work reported here is to observe the effects of chirality on spin textures, an objective that requires a lattice potential to be included in the model, hence demanding a two-dimensional lattice structure to be specified. Any periodic system in the $x-y$ plane can be constructed from one of the five two-dimensional Bravais lattices. The basis placed on each

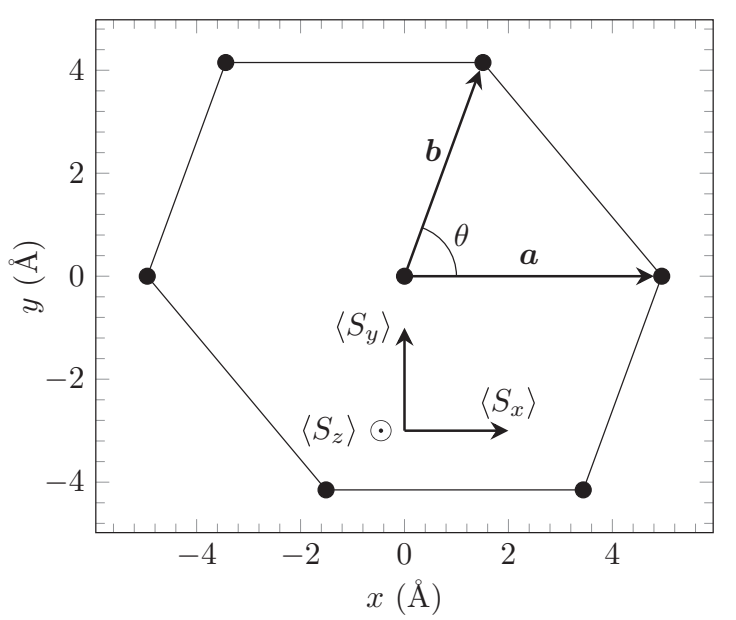

FIG. 1. Direct lattice points for an oblique lattice, where $\boldsymbol{a}$ and $\boldsymbol{b}$ are the primitive lattice vectors. $|\boldsymbol{a}|$ was set constant to $4.95 \AA$ for all calculations. The directions of the spin expectation values are shown relative to the real-space directions.

lattice site may be simply a single atom or a multi-atom cluster or molecule (which may itself be chiral). Of these Bravais lattices, only the so-called oblique lattice has no $(x \rightarrow-x)$ or $(y \rightarrow-y)$ reflection symmetries; all the others (rectangular, centered rectangular, square and hexagonal) possess these symmetries. Thus the oblique lattice is called a chiral system while the other four are achiral. In this paper, calculations were performed on the oblique lattice itself (not a multi-atom unit cell) as a function of its structural parameters without varying the potential parameters. This is because special values of these potential parameters correspond to a particular hexagonal lattice (see the following section). Varying the structural parameters continuously provides a convenient way of transforming an achiral system into a chiral system. In this way, the effects of chirality on the spin textures can be observed and investigated.

\section{COMPUTATIONAL DETAILS}

The input parameters used in the calculations reported below were based on the surface of $\mathrm{Bi} / \mathrm{Ag}(111)$ described in Ref. [24]. The Fourier components of the potential were assigned the values $V_{0}=-6.6 \mathrm{eV}$ and $V_{G}= \pm i 5 \mathrm{eV}$ which associate with $\boldsymbol{G}=0$ and the shortest reciprocal-lattice vectors, respectively. Except for $V_{\boldsymbol{G}=0}$, adjacent Fourier components were always of opposite sign such that all lattices had an antisymmetric potential that breaks local inversion symmetry [24]. This asymmetry is a consequence of the $A B C$ stacking present in face-centered cubic (111) surfaces which was retained through the achiral lattices modelled. The other parameters used were an effective electron mass of $-0.4 m_{e}$, a Rashba coefficient of $0.392 \mathrm{eV} \AA$ and a scaled in-plane potential gradient [24]. Tests confirming that the model correctly reproduces the work conducted by Premper et al. are shown in the supplementary information [27].

Figure 1 shows the direct lattice points of an oblique structure from which all reciprocal lattices and the corresponding reciprocal-lattice vectors $\boldsymbol{g}$ were derived. For arbitrary 
$\theta$ and $|\boldsymbol{a}| /|\boldsymbol{b}|$ the chirality of this structure is evident. The first calculation presented used the hexagonal structure of $\mathrm{Bi} / \mathrm{Ag}(111)$ produced by setting $|\boldsymbol{a}|=|\boldsymbol{b}|=4.95 \AA$ and $\theta=$ $60^{\circ}$ where $|\boldsymbol{b}|$ and $\theta$ are called the structural parameters. The results of this calculation were compared with that of a chiral lattice with the structural parameters $|\boldsymbol{b}|=|\boldsymbol{a}|-1 \AA$ and $\theta=60^{\circ}$. To then observe variations in the spin texture, different chiral lattices were used. These were obtained by varying the structural parameters: $|\boldsymbol{b}|$ was changed for constant $\theta=60^{\circ}$ and $\theta$ was varied for constant $|\boldsymbol{b}|=|\boldsymbol{a}|-1 \AA$. The complementary enantiomorph of a particular chiral lattice was generated by inverting the $\hat{\boldsymbol{x}}$ component for all lattice vectors.

The eigenvectors and eigenvalues reported in the next section were calculated as a function of $k_{x}$ for the states $\left|\Psi\left(k_{x}, k_{y}=0\right)\right\rangle$. In this case, the spin polarizations are labeled as longitudinal for $\left\langle S_{x}\right\rangle$, tangential for $\left\langle S_{y}\right\rangle$, and perpendicular for $\left\langle S_{z}\right\rangle$. The directions of the spin are indicated in Fig. 1. Calculations were also performed as a function of $k_{y}$ for the states $\left|\Psi\left(k_{x}=0, k_{y}\right)\right\rangle$ and the results of these are included where appropriate. For this state, the longitudinal spin direction is $\left\langle S_{y}\right\rangle$ and the tangential direction is $\left\langle S_{x}\right\rangle$.

\section{RESULTS}

Figures 2(a) and 2(b) show the lowest-energy eigenvalues and the spin expectation values of the "spin-up" band, for $\left|\Psi\left(k_{x}, k_{y}=0\right)\right\rangle$, of the achiral hexagonal lattice. The bands are labeled "spin up" and "spin down" because the signs of $\left\langle S_{y}\right\rangle$ are positive and negative, respectively. The main difference between the values shown in Fig. 2(b) and those of the original formulation of the Rashba effect is that $\left\langle S_{z}\right\rangle$ is not zero for all $k_{x}$. This is caused by the nonzero in-plane potential gradients. The longitudinal spin polarization $\left\langle S_{x}\right\rangle$ is observed to be zero for all $k_{x}$. This can be rationalised by considering the hexagonal lattice to be an equal mixture of both enantiomorphs. The size of the band splitting, $k_{0}$, in Fig. 2(a) agrees with that reported previously [6,24].

The lowest-energy eigenvalues for $\left|\Psi\left(k_{x}, k_{y}=0\right)\right\rangle$ of the chiral oblique lattice are shown in Fig. 2(c). The spin splitting is approximately twice as large as that in Fig. 2(a). Even though the chiral system lacks mirror symmetry, the bands show a symmetry about $k_{x}=0 \AA^{-1}$. This is because a rotation of $180^{\circ}$ about the reciprocal-lattice point at $(0,0)$ generates the equivalent chiral lattice.

The spin expectation values of the spin-up band in Fig. 2(c) are shown in Fig. 2(d). There are two important differences in the spin expectation values between the achiral [Fig. 2(b)] and chiral lattices [Fig. 2(d)]. First, there is an increase in the magnitude of $\left\langle S_{z}\right\rangle$ in the region $-0.5<k_{x}<0.5 \AA^{-1}$. This is a result of the in-plane components of the potential gradient and the chirality of the lattice. Second, there is a nonzero longitudinal spin polarization shown by $\left\langle S_{x}\right\rangle$ for the chiral lattice. It is positive, small in magnitude $(\sim 2.5 \%$ at its maximum), and varies as a function of $k$. This result is due to the oblique lattice lacking mirror symmetry and the perpendicular component of the potential gradient.

Figure 3 shows the longitudinal spin polarization as a function of $k_{x}$, where Fig. 3(a) shows $\left\langle S_{x}\right\rangle$ from Fig. 2(d) but with an increased resolution and Fig. 3(b) shows $\left\langle S_{x}\right\rangle$ for the

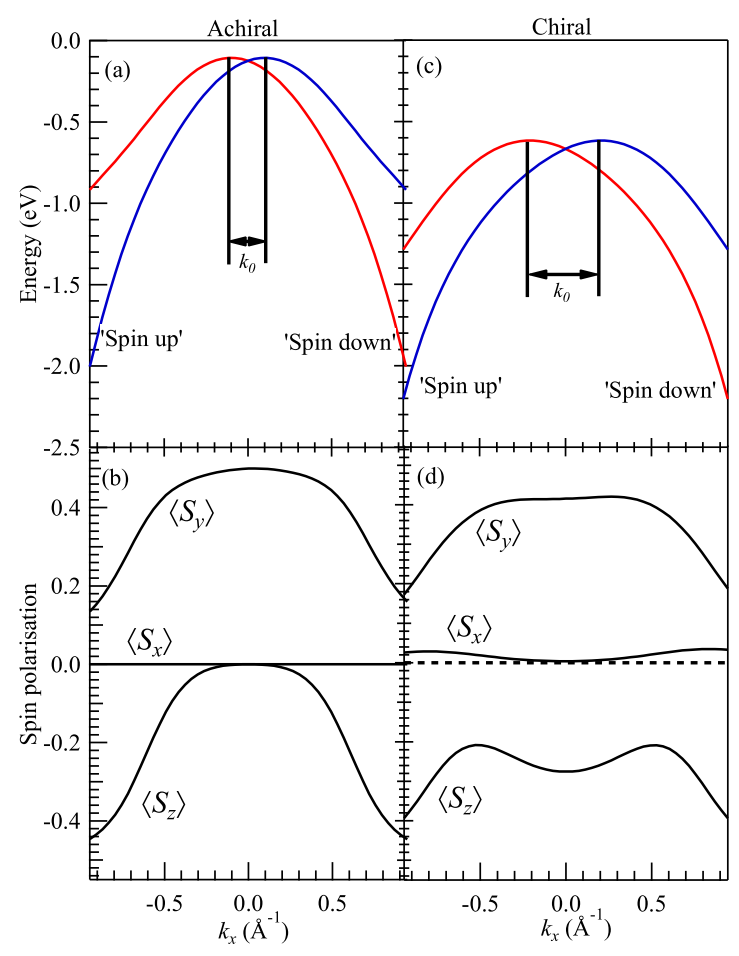

FIG. 2. (a) Lowest-energy eigenvalues for the states $\mid \Psi\left(k_{x}, k_{y}=\right.$ $0)\rangle$ of a hexagonal lattice where $k_{0}$ describes the Rashba splitting. Panel (c) shows the same eigenvalues for the oblique lattice. The first Brillouin-zone boundaries in the $k_{x}$ direction for the hexagonal and oblique lattices are at \pm 0.88 and $\pm 0.87 \AA^{-1}$, respectively (see supplementary information for an extended energy eigenvalue diagram). The spin expectation values of the spin-up band in panels (a) and (c) are shown in panels (b) and (d), respectively. The spin expectation values of the spin down were calculated and agree with Kramer's theorem [29] (not shown).

spin-up band of the complementary chiral lattice (the mirrorreflected lattice). Clearly, the longitudinal spin polarization is equal in magnitude but opposite in sign for the enantiomorphs. The other spin polarizations $\left\langle S_{y}\right\rangle$ and $\left\langle S_{z}\right\rangle$ remain unchanged

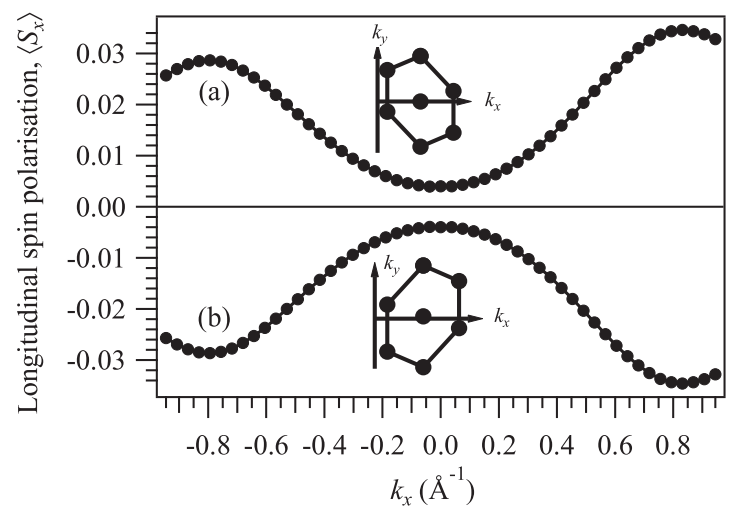

FIG. 3. Longitudinal spin polarization, $\left\langle S_{x}\right\rangle$, obtained from the spin-up band of the lowest-energy eigenvalues for the states $\left|\Psi\left(k_{x}, k_{y}=0\right)\right\rangle$. (a) Magnified image of $\left\langle S_{x}\right\rangle$ from Fig. 2(d) calculated by using the displayed upper reciprocal lattice, and (b) $\left\langle S_{x}\right\rangle$ for the mirror-reflected structure shown. 


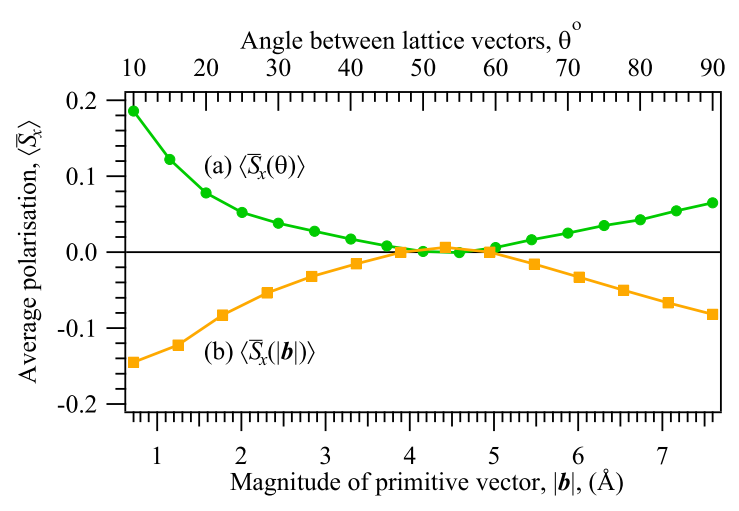

FIG. 4. The average longitudinal spin polarization as a function of (a) the angle between the primitive lattice vectors, and (b) the magnitude of the primitive lattice vector $|\boldsymbol{b}|$.

between the enantiomorphs. Spin expectation values were also calculated for the states $\left|\Psi\left(k_{x}=0, k_{y}\right)\right\rangle$ (i.e., with $\boldsymbol{k}$ along the $y$ direction), and these show that $\left\langle S_{y}\right\rangle$ inverts between the enantiomorphs whereas $\left\langle S_{x}\right\rangle$ does not. Furthermore, in contrast with the calculations associated with Fig. $3,\left\langle S_{z}\right\rangle$ is also found to invert between the enantiomorphs.

The influence of chirality on the spin polarizations is further revealed by varying the structural parameters and the magnitude of the perpendicular component of the potential gradient, $\partial_{z} V=E_{z}$. An average longitudinal spin polarization $\left\langle\bar{S}_{x}\right\rangle$ for the spin-up band (of the same enantiomorph) was calculated over the range $k_{y}=0$ to the first Brillouin-zone boundary for $\left|\Psi\left(k_{x}=0, k_{y}\right)\right\rangle$. This was performed by using oblique lattices with varying values of the structural parameters $|\boldsymbol{b}|$ and $\theta$ (see Sec. III).

Figure 4(a) shows the variation of the average longitudinal spin polarization against increasing $\theta$, and Fig. 4(b) shows the variation with the magnitude of the primitive-lattice vector, $|\boldsymbol{b}|$. Note that, for $|\boldsymbol{b}|=|\boldsymbol{a}|=4.95 \AA$ and $\theta=60^{\circ}$, the oblique lattice becomes hexagonal. Figures 4(a) and 4(b) thus show that the average longitudinal spin polarization increases as the values of either of the structural parameters diverge from their respective hexagonal structure values. The same calculation

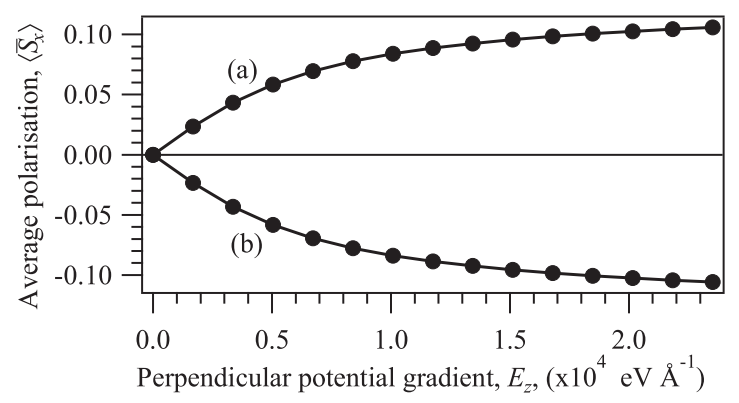

FIG. 5. Average longitudinal spin polarization as a function of the magnitude of the perpendicular component of the potential gradient. The average polarizations were calculated by using the same procedure as in Fig. 4. Panel (a) shows the dependence for the enantiomorph associated with Fig. 4, and panel (b) corresponds to the mirror-reflected lattice. was performed for the complementary enantiomorph and produced the same variation but inverted (not shown).

The variation of the average longitudinal spin polarization as a function of $E_{z}$ is displayed in Fig. 5. This shows that, for small $E_{z}$, the longitudinal spin polarization is proportional to the perpendicular potential gradient. Figure 5 also infers the relationship between the spin-orbit coupling and the longitudinal spin polarization. This is because the spin-orbit coupling is derived by using the perpendicular potential gradient such that, for a spherical potential, $E_{z} \propto Z / r^{2}$. Hence, increasing $E_{z}$ is equivalent to increasing the spin-orbit coupling (and the atomic number $Z$ ), which corresponds to an increased longitudinal spin polarization. In the next section, a heuristic model is put forward to understand this effect.

\section{DISCUSSION}

In the original Rashba model, where the in-plane electronic motion is approximated to be free and only the potential gradient is perpendicular to the plane, the spin-orbit interaction from Eq. (1) becomes $\alpha \boldsymbol{\sigma} \cdot\left(\boldsymbol{k}_{\|} \times \hat{z}\right)$. Thus the spin polarization is clearly tangential to the surface crystal momentum $k_{\|}$, and there is no longitudinal or perpendicular spin polarization. In contrast, when an in-plane potential is included, the calculations show nonzero longitudinal $\left\langle S_{x}\right\rangle$ and perpendicular $\left\langle S_{z}\right\rangle$ polarizations, which are shown in Figs. 2(b) and 2(d). Finite values of $\left\langle S_{z}\right\rangle$ arise because of the nonzero in-plane potential gradients and antisymmetric Fourier coefficients. However, the appearance of a longitudinal spin polarization is less intuitive. This longitudinal polarization can be understood in terms of the following heuristic model:

Consider the effective velocities for Bloch electrons in lattices of different symmetries. The traditional approach to electron dynamics $[29,30]$ associates this velocity $\boldsymbol{v}$ with the momentum derivative of the energy eigenfunctions $E(\boldsymbol{k})$,

$$
\boldsymbol{v}(\boldsymbol{k})=\frac{\partial E(\boldsymbol{k})}{\partial \boldsymbol{k}} .
$$

The energy contours shown in Fig. 6(a) indicate that for the hexagonal lattice this derivative is zero for $k_{x}=0$ and $k_{y}=$ 0 for all bands. However, this is not the case for the chiral lattice, as shown in Fig. 6(b). Furthermore, the momentumspace gradient of the energy eigenvalues in Eq. (5) are equal to momentum expectation values in the corresponding Bloch state [29]:

$$
\nabla_{\boldsymbol{k}} E(\boldsymbol{k})=\left(m^{*}\right)^{-1}\langle\Psi(\boldsymbol{k})|\hat{\boldsymbol{p}}| \Psi(\boldsymbol{k})\rangle .
$$

Thus, the calculated energy contours in Fig. 6(a) imply that a Bloch state $\left|\Psi\left(k_{x}, k_{y}=0\right)\right\rangle$ is associated with a velocity $\boldsymbol{v}\left(k_{x}, k_{y}=0\right)$ which is entirely along the $x$ direction for the hexagonal lattice, but has a component along the $y$ axis for the chiral lattice. Moreover, it can be shown analytically that for a lattice with mirror symmetry the momentum expectation value $\left\langle\Psi\left(k_{x}, k_{y}=0\right)\left|\hat{p}_{y}\right| \Psi\left(k_{x}, k_{y}=0\right)\right\rangle$ must always be zero. Hence, our calculations confirm the general results: for the chiral lattice,

$$
\begin{aligned}
& \left(m^{*}\right)^{-1}\left\langle\Psi\left(k_{x}, k_{y}=0\right)\left|\hat{p}_{y}\right| \Psi\left(k_{x}, k_{y}=0\right)\right\rangle \\
& \quad=v_{y}\left(k_{x}, k_{y}=0\right) \neq 0
\end{aligned}
$$




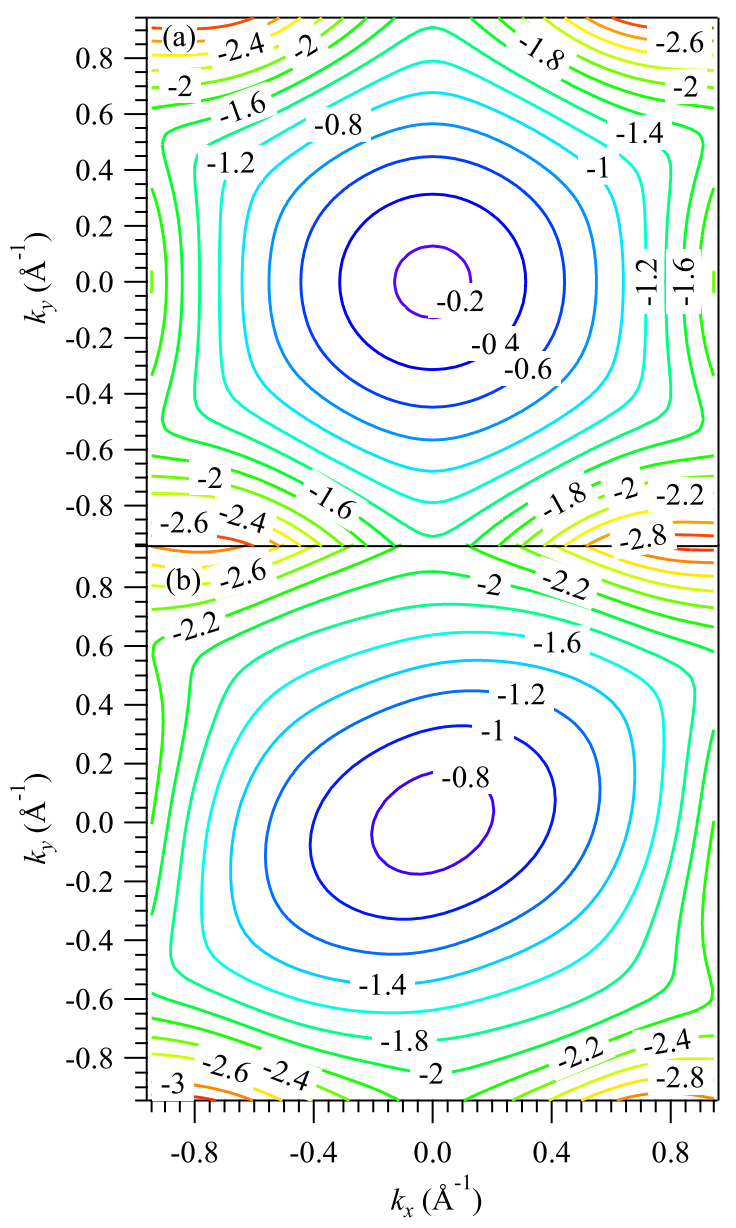

FIG. 6. Constant energy eigenvalues (in $\mathrm{eV}$ ) as a function of $k_{x}$ and $k_{y}$ for (a) the hexagonal lattice, and (b) one enantiomorph of the oblique lattice. These were obtained from the energy eigenvalues shown in Fig. 2.

and for the achiral lattice,

$$
\begin{aligned}
& \left(m^{*}\right)^{-1}\left\langle\Psi\left(k_{x}, k_{y}=0\right)\left|\hat{p}_{y}\right| \Psi\left(k_{x}, k_{y}=0\right)\right\rangle \\
& \quad=v_{y}\left(k_{x}, k_{y}=0\right)=0 .
\end{aligned}
$$

This velocity is now treated as a classical variable and a Lorentz transformation is performed from the laboratory frame to the rest frame of an electron in the Bloch state $\left|\Psi\left(k_{x}, k_{y}=0\right)\right\rangle$. This electron is taken to have a velocity $\boldsymbol{v}=c\left(\beta_{x}, \beta_{y}\right)$, where $\beta_{y}$ is nonzero only in a chiral system (and $c$ is the speed of light). In the laboratory frame, with no magnetic field present, there exists a static electric field $\boldsymbol{E}$ given by the potential gradient. In the electron's rest frame a magnetic field $\boldsymbol{B}^{\prime}$ appears [31] with the in-plane components

$$
B_{y}^{\prime}=\gamma \beta_{x} \frac{E_{z}}{c} \text { and } B_{x}^{\prime}= \pm \gamma \beta_{y} \frac{E_{z}}{c},
$$

where $\gamma=\left(1+\beta^{2}\right)^{-1 / 2}$ is the Lorentz factor. The electron spin will align with this magnetic field; this is the "semiclassical" picture of the origin of the spin-orbit interaction. The $B_{y}^{\prime}$ component shown in Eq. (9) is similar to the spin polarization calculated from the Rashba model; it is tangential to the nonzero momentum eigenket $\left|k_{x}, k_{y}=0\right\rangle$ and the perpendicular electric field. There is also a magnetic-field component along the $x$ direction in Eq. (9), so a finite longitudinal spin polarization is expected. The heuristic model proposed suggests that the longitudinal polarization shown in Fig. 2(d) is due to the chiral lattice lacking mirror symmetry which then results in a nonzero $\beta_{y}$ velocity determined by Eq. (7). The velocity of the electron in the $y$ direction, $\beta_{y}$, can be positive or negative such that $B_{x}^{\prime}$ displayed in Eq. (9) has been modified with a prefactor of \pm to represent the handedness of the enantiomorphs. Figure 3 shows that the longitudinal spin polarization does invert between the two enantiomorphs.

Equation (9) also suggests that the longitudinal spin polarization should be proportional to both the velocity $\beta_{y}$, and the perpendicular electric field $E_{z}$ for small $B_{x}^{\prime}$. The velocity $\beta_{y}$ depends on the structural parameters $\theta$ and $|\boldsymbol{b}|$ shown in Fig. 1 because these parameters allow the lattice to transform from achiral to chiral. Figure 4(a) shows that, at $\theta=60^{\circ}$, the spin polarization is nominally zero because the lattice points are approximately hexagonal as $|\boldsymbol{b}|=|\boldsymbol{a}|-1 \AA$. Therefore, as $\theta$ departs from $60^{\circ}$ the longitudinal spin polarization increases because the structure is distorted further from the hexagonal symmetry. Similarly, the same relationship is observed when the magnitude of the basis vector, $|\boldsymbol{b}|$, is varied as shown in Fig. 4(b). At $|\boldsymbol{b}|=4.95 \AA$ the lattice is hexagonal and the longitudinal spin polarization is zero. As $|\boldsymbol{b}|$ is varied from $4.95 \AA$ the longitudinal spin polarization increases. Equation (9) shows that the longitudinal spin polarization is zero for $E_{z}=0$ (because $B_{x}^{\prime}=0$ ) and is expected to evolve linearly as $E_{z}$ increases. These effects are both observed from the initial three points shown in Fig. 5.

When the structural distortion of the chiral lattice from hexagonal becomes large, the longitudinal spin polarization is no longer a linear function of $\beta_{y}$. Similarly, when the perpendicular electric field $E_{z}$ becomes large, deviations are found (see Fig. 5) from the linear relationship in Eq. (9). Such detailed behavior would presumably be reproduced by modern ab initio methods such as relativistic-DFT calculations, but are beyond this heuristic model.

The perpendicular spin expectation values $\left\langle S_{z}\right\rangle$ shown in Figs. 2(b) and 2(d) are also explained by the heuristic model introduced above. Using the same derivation that produced the in-plane magnetic-field components displayed in Eq. (9), the perpendicular component is [31]

$$
B_{z}^{\prime}=-\frac{\gamma}{c}\left(\beta_{x} E_{y}-\beta_{y} E_{x}\right)
$$

where $E_{x}$ and $E_{y}$ are the in-plane electric fields. Furthermore, for an electron associated with $\left|\Psi\left(k_{x}, k_{y}=0\right)\right\rangle$, the chiral contribution comes from the term $\beta_{y} E_{x}$, since $\beta_{y} \neq 0$ for the chiral lattice. Similarly, for $\left|\Psi\left(k_{x}=0, k_{y}\right)\right\rangle$ the chiral contribution is from $\beta_{x} E_{y}$. This implies that $\left\langle S_{z}\right\rangle$ for the states $\left|\Psi\left(k_{x}, k_{y}=0\right)\right\rangle$ of the chiral lattice has no chiral contribution because $E_{x}$ is zero (see the supplementary information [27]). Therefore, $\left\langle S_{z}\right\rangle$ does not change between the two enantiomorphs as obtained from the calculations. However, for $\left|\Psi\left(k_{x}=0, k_{y}\right)\right\rangle$ the values of $\left\langle S_{z}\right\rangle$ were found to invert between the two enantiomorphs. This is because the only non-negligible term [27] contributing to the perpendicular magnetic field comes from $\beta_{x} E_{y}$, which is the chiral factor. For a more general chiral lattice $\left\langle S_{z}\right\rangle$ is different between the enantiomorphs because both terms in Eq. (10) will be nonzero. 
The nonzero perpendicular spin polarization shown in Fig. 2(d) at $k_{x}=0$ which is zero in Fig. 2(b) is another consequence of the lack of mirror symmetry of the oblique lattice. To see this, Eq. (8) is rewritten by using $\hat{p}_{x}$ to produce the equivalent equality for the achiral lattice

$$
\left\langle\Psi\left(k_{x}=0, k_{y}\left|\hat{p}_{x}\right| \Psi\left(k_{x}=0, k_{y}\right)\right\rangle=0,\right.
$$

which is true for any $k_{y}$ including $k_{y}=0$. This implies that $\beta_{x}=0$ at $k_{x}=0$ for an achiral lattice but not in general for the chiral lattice. The maximum in absolute value of $\left\langle S_{z}\right\rangle$ shown in Fig. 2(d) at $k_{x}=0$ is a result of the in-plane electric field $E_{y}$ (the functional form of which is derived in the supplementary information [27]). Therefore, at $k_{x}=k_{y}=0$ the magnitude of the electric field is maximized, resulting in a large perpendicular spin polarization component.

Figures 2(b) and 2(d) show that $\left\langle S_{y}\right\rangle \neq 0$ at $k_{x}=k_{y}=0$ even though Eq. (9) shows that $B_{y} \propto \beta_{x}$. This can be explained by noticing that the eigenfunctions derived from either the simple Rashba model or the full calculations, which include an in-plane potential, contain a phase that is absent from the semiclassical heuristic model described in this section. This phase produces the tangential spin components [22].

Experimental observation of the longitudinal spin polarization should be possible by using spin-resolved photoemission [32-34], as several different types of chiral surfaces exist [35]. Such experiments are to be distinguished from spin-resolved photoemission experiments where the chirality is a result of the experimental geometry, as explored in Ref. [36]. The key role of the spin-orbit interaction implies that surfaces composed of heavy atoms are expected to produce a significant longitudinal spin polarization. To determine whether the longitudinal spin polarization is energetically resolvable in spin-resolved photoemission experiments, the above heuristic model can be used to calculate an energy shift $\Delta E$ associated with the longitudinal polarization and its corresponding magnetic-field component $B_{\text {long }}$. This energy shift is obtained from $\Delta E=g_{s} \mu_{B} B_{\text {long }}$, where $\mu_{B}$ is the Bohr magneton and $g_{s}$ is the electron $g$ factor. By using the Rashba parameter appropriate for $\mathrm{Bi} / \mathrm{Ag}(111)$ and a momentum expectation value associated with the longitudinal spin polarization, an energy shift of $24 \mathrm{meV}$ is obtained. Below $100 \mathrm{~K}$, thermal fluctuations are an insignificant factor in randomizing electron spins with respect to the longitudinal direction and, because the total instrumental resolution of state-of-the-art spin-resolved photoemission experiments at synchrotrons is $\sim 70 \mathrm{meV}$ [37] or better [38], this will allow for the polarization of the surface states to be resolved in the future.

\section{CONCLUSION}

The Rashba model has been generalized, extending the approach of Premper et al., to include lattices of different symmetries, the lattice potential and its in-plane gradients. This was done by using a basis of spin and momentum eigenkets, and a potential described by a set of Fourier components. The in-plane potential gradients included in the spin-orbit interaction were derived from these parameters. The perpendicular component was approximated to a constant as performed in the Rashba model. This parametrized model was solved numerically by standard linear algebra techniques to derive the energy eigenfunctions, eigenvectors, and the spin expectation values.

The main focus of this paper has been to use this parametrized model to investigate the effects of lattice symmetry on the spin-dependent electronic structure of the system. A simple two-dimensional chiral lattice was chosen for study, the oblique Bravais lattice, which has no mirror symmetries except for special values of its basis vectors when it becomes, for example, the hexagonal Bravais lattice. The calculations show that chirality is associated with the appearance of a longitudinal spin polarization and modifications of the perpendicular polarization.

To provide physical insight into these findings, a heuristic model has been suggested by utilizing a semiclassical approach to electron dynamics: a velocity is associated with a given Bloch state and is then used in a Lorentz transformation. This heuristic model explains key differences between the behavior of chiral and achiral systems, and serves as a guide to observations, experimental and theoretical, on more complex systems.

\section{ACKNOWLEDGMENTS}

This work was supported by EPSRC (UK) under Grant No. EP/M507969/1. Funding was also received from ASTeC and the Cockcroft Institute (UK) The data associated with the paper are openly available from Mendeley: http://dx.doi.org/10.17632/sjvrp2ft2x.1.
[1] V. Sunko, H. Rosner, P. Kushwaha, S. Khim, F. Mazzola, L. Bawden, O. J. Clark, J. M. Riley, D. Kasinathan, M. W. Haverkort et al., Nature (London) 549, 492 (2017).

[2] J. M. Riley, F. Mazzola, M. Dendizk, M. Michiardi, T. Takayama, L. Bawden, C. Granerød, M. Leandersson, T. Balasubramanian, M. Hoesch et al., Nat. Phys. 10, 835 (2014).

[3] E. A. Seddon, in Handbook of Spintronics, edited by Y. Xu, D. D. Awschalom, and J. Nitta (Springer, Netherlands, 2016), Chap. 22.

[4] T. Okuda, J. Phys.: Condens. Matter 29, 483001 (2017).

[5] S. LaShell, B. A. McDougall, and E. Jensen, Phys. Rev. Lett. 77, 3419 (1996).
[6] C. R. Ast, J. Henk, A. Ernst, L. Moreschini, M. C. Falub, D. Pacilé, P. Bruno, K. Kern, and M. Grioni, Phys. Rev. Lett. 98, 186807 (2007).

[7] C. R. Ast, D. Pacilé, L. Moreschini, M. C. Falub, M. Papagno, K. Kern, M. Grioni, J. Henk, A. Ernst, S. Ostanin, and P. Bruno, Phys. Rev. B 77, 081407(R) (2008).

[8] I. Gierz, T. Suzuki, E. Frantzeskakis, S. Pons, S. Ostanin, A. Ernst, J. Henk, M. Grioni, K. Kern, and C. R. Ast, Phys. Rev. Lett. 103, 046803 (2009).

[9] K. Sakamoto, H. Kakuta, K. Sugawara, K. Miyamoto, A. Kimura, T. Kuzumaki, N. Ueno, E. Annese, J. Fujii, A. Kodama et al., Phys. Rev. Lett. 103, 156801 (2009). 
[10] K. Ishizaka, M. S. Bahramy, H. Murakawa, M. Sakano, T. Shimojima, T. Sonobe, K. Koizumi, S. Shin, H. Miyahara, A. Kimura et al., Nat. Mater. 10, 521 (2011).

[11] K. F. Mak, K. He, J. Shan, and T. F. Heinz, Nat. Nanotechnol. 7, 494 (2012).

[12] R. Bertoni, C. W. Nicholson, L. Waldecker, H. Hübener, C. Monney, U. D. Giovannini, M. Puppin, M. Hoesch, E. Springate, R. T. Chapman et al., Phys. Rev. Lett. 117, 277201 (2016).

[13] Z. Y. Zhu, Y. C. Cheng, and U. Schwingenschlögl, Phys. Rev. B 84, 153402 (2011).

[14] A. Dankert and S. P. Dash, Nat. Commun. 8, 16093 (2017).

[15] S.-T. Lo, C.-H. Chen, J.-C. Fan, L. W. Smith, G. L. Creeth, C.-W. Chang, M. Pepper, J. P. Griffiths, I. Farrer, H. E. Beere et al., Nat. Commun. 8, 15997 (2017).

[16] S. Mayer, C. Nolting, and J. Kessler, J. Phys. B: At. Mol. Opt. Phys. 29, 3497 (1996).

[17] S. G. Ray, S. S. Daube, G. Leitus, Z. Vager, and R. Naaman, Phys. Rev. Lett. 96, 036101 (2006).

[18] B. Göhler, V. Hamelbeck, T. Z. Markus, M. Kettner, G. F. Hanne, Z. Vager, R. Naaman, and H. Zacharias, Science 331, 894 (2011).

[19] J. Gersten, K. Kaasbjerg, and A. Nitzan, J. Chem. Phys. 139, 114111 (2013).

[20] P. S. Farago, J. Phys. B: At. Mol. Phys. 13, L567 (1980).

[21] N. A. Cherepkov, J. Phys. B: At. Mol. Phys. 14, L623 (1981).

[22] A. Manchon, H. C. Koo, J. Nitta, S. M. Frolov, and R. A. Duine, Nat. Mater. 14, 871 (2015).

[23] R. M. Martin, Electronic Structure: Basic Theory and Practical Methods (Cambridge University Press, Cambridge, 2008).

[24] J. Premper, M. Trautmann, J. Henk, and P. Bruno, Phys. Rev. B 76, 073310 (2007).

[25] L. Fu, Phys. Rev. Lett. 103, 266801 (2009).
[26] This form emits the Darwin and mass-velocity terms, hence in general it is not Hermitian. This is accounted for in the numerical calculations.

[27] See Supplemental Material at http://link.aps.org/supplemental/ 10.1103/PhysRevB.97.235427 for the modelling of Bi/Ag(111), the derivation of the in-plane potential gradients and the extended energy eigenvalue diagram.

[28] W. H. Press, S. A. Teukolsky, W. T. Vetterling, and B. P. Flannery, Numerical Recipes in $\mathrm{C}++$, 2nd ed. (Cambridge University Press, Cambridge, 2002).

[29] N. W. Ashcroft and N. D. Mermin, Solid State Physics (Saunders, Philadelphia, 1976).

[30] J. M. Ziman, Principles of the Theory of Solids, 2nd ed. (Cambridge University Press, Cambridge, 1972).

[31] J. D. Jackson, Classical Electrodynamics, 3rd ed. (John Wiley and Sons, Inc., New York, 1999).

[32] C. H. Park and S. G. Louie, Phys. Rev. Lett. 109, 097601 (2012).

[33] J. Sánchez-Barriga, A. Varykhalov, J. Braun, S.-Y. Xu, N. Alidoust, O. Kornilov, J. Minár, K. Hummer, G. Springholz, G. Bauer et al., Phys. Rev. X 4, 011046 (2014).

[34] Z. H. Pan, E. Vescovo, A. V. Fedorov, D. Gardner, Y. S. Lee, S. Chu, G. D. Gu, and T. Valla, Phys. Rev. Lett. 106, 257004 (2011).

[35] S. J. Jenkins, Chirality at Solid Surfaces (John Wiley and Sons, New York, 2018).

[36] K. Kobayashi, K. Yaji, K. Kuroda, and F. Komori, Phys. Rev. B 95, 205436 (2017).

[37] C. Bigi, P. K. Das, D. Benedetti, F. Salvador, D. Krizmancic, R. Sergo, A. Martin, G. Panaccione, G. Rossi, J. Fujii, and I. Vobornik, J. Synchrotron Rad. 24, 750 (2017).

[38] T. Okuda, K. Miyamaoto, H. Miyahara, K. Kuroda, A. Kimura, H. Namatame, and M. Taniguchi, Rev. Sci. Instrum. 82, 103302 (2011). 